\title{
Management of non-visualization following dynamic sentinel lymph node biopsy for squamous cell carcinoma of the penis \\ Authors
}

Varun Sahdev,

Department of Urology, University College London

Hospitals NHS Foundation Trust, London, UK

Maarten Albersen,

Department of Urology, University College London

Hospitals NHS Foundation Trust, London, UK

University Hospitals Leuven, Leuven, Belgium

Michelle Christodoulidou,

Department of Urology, University College London

Hospitals NHS Foundation Trust, London, UK

Arie Parnham,

Department of Urology, University College London

Hospitals NHS Foundation Trust, London, UK

Peter Malone,

Department of Urology, University College London

Hospitals NHS Foundation Trust, London, UK

Raj Nigam,

Department of Urology, University College London

Hospitals NHS Foundation Trust, London, UK

Jamshed Bomanji,

Institute of Nuclear Medicine, University College London

Hospital, London, UK

\section{Asif Muneer Corresponding author}

E-mail address: asif.muneer@uclh.nhs.uk

Department of Urology and NIHR Biomedical Research

Centre, University College London Hospitals NHS

Foundation Trust, London, UK

Correspondence: Asif Muneer, Department of Urology,

University College London Hospitals NHS Foundation

Trust, 235 Euston Road, NW12BU London, UK.e-mail: 
First published: 21 December 2016

DOI: $10.1111 /$ bju. 13680

\section{Abstract}

\section{Objectives}

To review the management and clinical outcomes of unior bilateral non-visualization of inguinal lymph nodes during dynamic sentinel lymph node biopsy (DSNB) in patients diagnosed with penile cancer and clinically impalpable inguinal lymph nodes (cN0), and to develop an algorithm for the management of patients in which nonvisualization occurs.

\section{Patients and Methods}

This is a retrospective observational study over a period of 4 years, comprising 166 patients with penile squamous cell carcinoma undergoing DSNB and followed up for a minimum of 6 months. All cases diagnosed with uni- or bilateral non-visualization of sentinel nodes in this cohort were identified from a penile cancer database. The management of the inguinal lymph nodes after nonvisualization and the oncological outcomes including local and regional recurrence rates were documented.

\section{Results}

Out of 166 consecutive patients undergoing DSNB, 20 patients (12\%) had unilateral non-visualization after injection of intradermal ${ }^{99 m} \mathrm{TC}$. Of these 20 patients, seven underwent repeat DSNB at a later date, with six having successful visualization. One patient had persistent nonvisualization and proceeded to a superficial modified inguinal lymphadenectomy (SML). None of these patients experienced recurrence at follow-up. A further seven 
patients underwent modified SML with on-table frozensection analysis of the lymph node packet; none of these patients were found to have micrometastatic disease in the inguinal lymph nodes, although one patient developed metastatic inguinal node disease at a later date. Six patients elected to undergo clinical surveillance and have remained disease-free.

\section{Conclusion}

Patients with impalpable inguinal lymph nodes undergoing DSNB with $\geq G 2 T 1$ disease should ideally have bilateral visualization of the sentinel lymph nodes, reflecting the drainage pattern from the primary tumour. In the present series, $12 \%$ of patients were found to have unilateral nonvisualization after DSNB. Among patients offered a repeat DSNB at a later date, localizing the sentinel node was successful in $86 \%$ of cases. Patients with favourable histological characteristics can be placed on clinical surveillance. Those with high-risk disease can be offered a repeat DSNB procedure on the proviso that SML may be carried out if there is repeated non-visualization. Larger cohorts are required to validate this proposed algorithm.

\section{Introduction}

Penile squamous cell carcinoma is a rare disease in Europe and North America, with an incidence of 1.5 per 100000 males [1, 2]. The management of the primary lesion is surgical resection; the surgical procedure performed depends on the site, degree of invasion and size of the lesion. Currently, the majority of cases are managed by performing penile-preserving procedures, which have a local recurrence rate of $4 \%$ at 5 years [3]; however, local recurrence does not appear to change the long-term prognosis as a further resection of the tumour can be performed. Despite efforts to explore alternative 
prognostic indicators over the past decade, the presence of metastatic disease in the inguinal lymph nodes remains the most important prognostic indicator [4-12].

It has been established by lymphangiography and cadaveric studies that lymphatic drainage from the primary penile tumour occurs bilaterally in a step-wise fashion.

This occurs initially to the superficial inguinal lymph nodes followed by the deep inguinal lymph nodes, and then to the ipsilateral pelvic lymph nodes [4-12].

Patients with clinically impalpable lymph nodes in the groins (cNO disease) have up to a $25 \%$ chance of harbouring occult metastases within the inguinal lymph nodes [4]. Offering all patients with cNO disease open radical inguinal lymphadenectomy or the less morbid superficial modified lymphadenectomy (SML) is known to be effective from an oncological perspective [13]; however, these procedures carry a morbidity rate of up to $70 \%$ and are deemed unnecessary in $75-80 \%$ of patients with cNO disease [14].

In 1977, Cabanas [15] described the use of sentinel node biopsy for the assessment of inguinal lymph nodes in patients with penile cancer based on lymphangiograms, the premise being that patients with a negative sentinel node biopsy would be spared the more invasive and morbid radical inguinal lymphadenectomy procedure. Only those patients found to have metastatic disease after sentinel node biopsy would have to undergo a radical inguinal lymphadenectomy. Subsequent technical refinements included the addition of technetium-99mlabelled nano-colloid and a blue dye, together with preoperative ultrasonography with fine-needle aspiration of morphologically abnormal lymph nodes. These additions have increased the sensitivity and specificity whilst maintaining a relatively low morbidity $(93-95 \%$, $100 \%,<5 \%$ respectively) [16-18].

According to the European Association of Urology 
guidelines, all patients with cNO disease and disease $>\mathrm{G} 1$ T1 should undergo SML or dynamic sentinel lymph node biopsy (DSNB) to ensure that micrometastatic disease in the inguinal nodes has been detected [4].

The aim of the present study was to review the management and clinical outcomes and identify any risk factors predisposing to non-visualization after DSNB, as well as to develop an algorithm for patients with nonvisualization.

\section{Patients and Methods}

\section{Patients}

A total of 166 patients diagnosed with penile squamous cell carcinoma ( $\geq \mathrm{G} 2 \mathrm{~T} 1$ ) and cN0 disease between March 2010 and July 2016 underwent DSNB. The images and surgical case notes were reviewed and the long-term follow-up recorded in a penile cancer database.

\section{Dynamic Sentinel Node Biopsy Protocol}

On the morning of the surgery, radiolabelled $99 \mathrm{~m}$ Tc was injected intra-dermally at four quadrants, either just proximal to where the tumour was located if already removed or around the tumour if DSNB was performed simultaneously with primary tumour resection. The tracer was administered at a dose of $30-40 \mathrm{MBq}$ in a total volume of $0.4 \mathrm{~mL}$.

As previously described, planar lymphoscintigraphy was carried out with a dynamic acquisition at $90 \times 10$-s frames performed immediately after injection [19]. An early static image was acquired at 20 min after the dynamic scan and again at $120 \mathrm{~min}$.

After $90 \mathrm{~min}, \mathrm{CT}$ images were obtained using conventional and combined single-photon emission CT, (SPECT)/CT. The SPECT/CT system consists of a dual-head, variableangle gamma camera, equipped with low-energy high- 
resolution collimators and spiral CT optimized for rapid rotation. SPECT acquisition (matrix $128 \times 128 ; 120$ frames; $15 \mathrm{~s}$ per frame) was performed with $3^{\circ}$-angle steps. The CT settings were $100 \mathrm{kV}$ and $\mathrm{mA}$ modulation. During reconstruction, SPECT images were corrected for attenuation and scatter. Both SPECT and CT axial 1.25$\mathrm{mm}$ slices were generated with the Xeleris application package (GE Healthcare, Little Chalfont, UK). The slices were transferred to an imaging viewing system (PACS) after generation of Digital Imaging and Communications in Medicine (DICOM) files. Fusion of images was performed with an Osirix DICOM viewer in a Unix-based operating system. All scans were evaluated and reported by nuclear medicine physicians preoperatively, and the site of the sentinel lymph node was marked on the patient's skin. Typical SPECT/CT images are shown in Figure 1 with bilateral $(A)$ and unilateral visualisation $(B)$.

\section{Figure 1.}

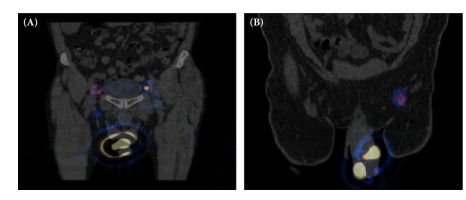

Single-photon emission CT/CT images showing (A) bilateral visualization and (B) unilateral visualization.

\section{Management of Patients with Non-Visualization}

In cases of non-visualization after the delayed static images, either a further $99 \mathrm{mTc}$ dose was injected intradermally with further delayed images or the patient underwent unilateral exploration of the visualized side with on-table assessment of the non-visualized side for gamma probe activity before considering an exploration. If there was no significant gamma probe activity, the groin was not explored. Non-significant gamma probe activity was defined as activity at skin level less than or equal to background count. The images were checked by a second 
nuclear medicine physician to exclude any error in the initial reports.

Where no exploration took place, patients were offered either a further DSNB procedure after 4-6 weeks, using an identical protocol with no dose escalation combined with the option of performing SML and frozen-section analysis on the same day. Patients were also offered close clinical and ultrasonographic surveillance as an alternative if the primary tumour was G2 with no evidence of lymphovascular or perineural invasion. This decision was based on both the grade and stage of the primary tumour and after discussion with the patient and careful explanation of the risks and benefits. All cases were discussed at a multidisciplinary team meeting.

We categorized the patients into three groups according to their management after non-visualization. Group A consisted of patients who underwent a further DSNB 4-6 weeks later; group B consisted of those who underwent superficial modified inguinal lymphadenectomy and group C underwent clinical surveillance as opposed to surgical intervention (Fig. 2).

\section{Figure 2.}

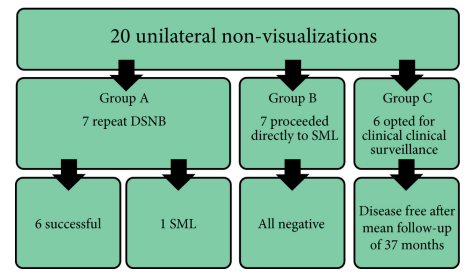

Flowchart outlining the patient pathway following unilateral non-visualization.

\section{Results}

In the cohort of 166 patients (median age 65.5, interquartile range $50-67)$, 20 patients (12\%) were found to have unilateral non-visualization, 10 (50\%) on the right side and $10(50 \%)$ on the left. There were no cases of bilateral non-visualization in our cohort.

\section{Group A}


Seven patients (35\%) underwent a repeat DSNB procedure at a later date, the majority of whom harboured grade 3 or pT2 disease (Table 1). Six of these (86\%) were found to have successful visualization on repeat DSNB.

Two patients had a previous inguinal hernia repair on the side of the non-visualization, which may have contributed to the initial non-visualization. There was no local or regional recurrence in this patient cohort, with a mean follow-up of 34.2 months (median [range] 29 [16-50 months] Table 1).

Table 1. Histological findings for patients in Group A who underwent repeat dynamic sentinel lymph node biopsy

\begin{tabular}{|l|l}
\hline Patient & $\begin{array}{l}\text { Histology from repeat DSNB: number positive lymph } \\
\text { nodes/number of lymph nodes taken }\end{array}$ \\
\hline 1 & $0 / 1$ \\
2 & $0 / 1$ \\
3 & $0 / 2$ \\
4 & $0 / 1$ \\
5 & $0 / 3$ \\
6 & $0 / 1$ \\
7 & $0 / 10^{*}$ \\
\hline
\end{tabular}

The one patient with non-visualization on a repeat DSNB procedure on the left side proceeded to SML during the same operation, which revealed no metastatic disease in the context of G3 pT1 disease of the foreskin. After a follow-up of 63 months there was no evidence of regional recurrence.

\section{Group B}

Seven patients underwent SML with frozen-section analysis; again, the majority of these patients had primary lesions, which were G3 T1 disease (Table 2). None of these patients had any evidence of metastatic squamous cell carcinoma in the excised lymph nodes. These seven patients had a mean (range) follow-up of 53 (25-66) months. One patient developed metastatic inguinal lymph nodes 4 months after a negative SML (0/4 lymph nodes 
analysed on the initial histology). Subsequent completion radical lymph node dissection showed $2 / 7$ metastatic lymph nodes. An ipsilateral pelvic lymphadenectomy revealed no further metastatic disease and the patient had no signs of recurrence at 36 months' follow-up.

Table 2. Histological findings for patients in Group B who proceeded directly to superficial modified lymphadenectomy with frozen section

\begin{tabular}{|l|l}
\hline Patient & $\begin{array}{l}\text { Histology from SML with frozen section: number positive lymph } \\
\text { nodes/number of lymph nodes taken }\end{array}$ \\
\hline 1 & $0 / 6$ (small lymphocytic lymphoma) \\
2 & $0 / 9$ (progressed to metastatic inguinal disease and underwent RLND) \\
3 & $0 / 15$ \\
4 & $0 / 7$ \\
5 & $0 / 16$ \\
6 & $0 / 9$ \\
7 & $0 / 10$ \\
\hline
\end{tabular}

One patient was found to have small lymphocytic lymphoma in his lymph nodes on histology and was referred to the haematology team. The presence of underlying lymphoma in this case might have contributed to non-visualization.

\section{Group C}

Six patients opted for clinical surveillance as opposed to further surgery. Four of these six had pT1 G2 disease.

Two out of six with higher grade or stage disease opted for surveillance despite the multidisciplinary team recommendation for a repeat surgical procedure. These were followed up with bilateral groin ultrasonography and clinical examination on a 3-4-monthly basis for the first 2 years and 6-monthly for year 3 , followed by clinical examination. All patients remain disease-free after a mean (median; range) follow-up of 41.5 (33; 19-62) months. These results are summarized in Table 3.

Table 3. Histological findings for patients Group C who 
elected to undergo clinical surveillance

\begin{tabular}{|l|ll}
\hline Patient & Histology of primary tumour and procedure \\
\hline 1 & G3 pT2a & Glansectomy \\
2 & G2 pT1 & WLE \\
3 & G2 pT1a & Circumcision \\
4 & G2/3 pT2 & Glansectomy \\
5 & G2 pT1 & WLE \\
6 & G1 pT2 & WLE \\
\hline
\end{tabular}

\section{Discussion}

The management of patients with penile SCC and cNO disease aims to minimize the morbidity associated with conventional inguinal lymphadenectomy but also detects the $15-25 \%$ of patients with occult disease. Patients with intermediate- or high-risk penile cancer and cNO disease can be offered DSNB, which should visualize the sentinel lymph node in both groins in the majority of cases [20]. DSNB is a technique based on the assumption that the lymphatic drainage from the penile cancer occurs bilaterally; however, despite the concomitant use of radioisotope and blue dye (Patente Bleu $\mathrm{V}$ ), unilateral, and more uncommonly bilateral, non-visualization has been previously reported in $19 \%$ and $2 \%$ of patients, respectively, on preoperative dynamic lymphoscintigraphy [21]. When encountering a non-visualization case, the alternative options are either to perform a more extensive procedure (superficial modified inguinal lymphadenectomy) or to place the patient on strict clinical and ultrasonographic surveillance.

The present study found that the rate of unilateral nonvisualization occurred in $12 \%$ of patients, which is slightly lower than rates in other studies (Table 4). From the results of this study we have proposed an algorithm to manage non-visualisation of sentinel nodes (Fig. 3).

Table 4. Incidence of non-visualization in previous studies Author/year Cohort Groin Unilateral non-visualization, Bilater: 


\begin{tabular}{|l|l|l|l|l}
\hline & size & s & $\boldsymbol{n}(\%)$ & patient \\
\hline $\begin{array}{l}\text { Sahdev et al. (present } \\
\text { study) }\end{array}$ & 166 & 332 & $20(12)$ & 0 \\
$\begin{array}{l}\text { Kroon et al. (2005) [18] } \\
\text { Kirrander et al. (2013) }\end{array}$ & 55 & 246 & $23(19)$ & $2(2)$ \\
{$[27]$} & 111 & $24(44)$ & $1(0.2)$ \\
\hline
\end{tabular}

\section{Figure 3.}

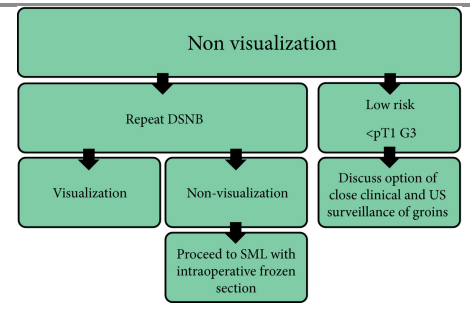

Proposed management algorithm for non-visualization at DSNB for penile cancer. LVI, lymphovascular invasion; US, ultrasound; SML, superficial modified lymphadenectomy.

The underlying causes of non-visualization remain unclear, but tumour occlusion of the lymphatics or the radioisotope dose, have been suggested as factors [16]. Tumour occlusion of the lymphatics was also reported as a possible cause for non-visualization by Kroon et al. [21], who explored the rate of non-visualization in a total of 246 groins. Non-visualization occurred in $11 \%$ of groins $(21 \%$ of patients), with unilateral non-visualization occurring in 23 patients (19\%) and bilateral non-visualization in two patients (2\%) [21]. Both patients who had bilateral nonvisualization were found to have significant penile oedema related to the primary tumour, and it was hypothesized that the lymphatic drainage was impaired secondary to this. Both of these patients remained on clinical surveillance and were disease-free on follow-up [21]. None of our patients had bilateral non-visualization, and only one patient was found to have metastatic inguinal lymph node disease 4 months after SML. It is difficult therefore to confirm Kroon's conclusion from our data. Another predictor for non-visualization was related to the dosage of $99 \mathrm{mTc}$ [16]. It is suggested that, for optimal results, it was important that the administered dose is at 
least $60 \mathrm{MBq}$. In breast cancer studies with nonvisualization, the dosage was also a predictor of successful visualization [22-26]. In our protocol, the $99 \mathrm{mTc}$ dosage was between 40 and $50 \mathrm{MBq}$, which is the standard dose for DSNB.

In the present series, the identification of two patients with previous surgery (hernia repair) and one patient with lymphoma, supports the theory that blocked or disrupted lymphatics may play a role in non-visualization at the time of DSNB; however, the number of events in this cohort did not allow statistical analysis to assess predictive factors for non-visualization.

Another plausible explanation may relate to the anatomy of the lymphatics draining the tumour, whereby there may be preferential lymphatic channels draining the penis to one side. Once this sentinel node is removed and the channel is disrupted, bilateral drainage may resume. This may explain the reason as to why the repeat DSNB procedures were successful.

The present data have shown that a repeat DSNB was successful in localizing the sentinel lymph node in $86 \%$ of cases and therefore provides an acceptable option for patients who have non-visualization; however, histological analysis of the sentinel nodes, after repeat DSNB, did not demonstrate the presence of metastatic disease. Similarly, of the seven patients in Group B, who proceeded directly to SML after initial non-visualization, none were found to have metastatic disease in any of the excised lymph nodes. In the one patient that developed a late recurrence, four lymph nodes were removed at the time of the SML, of which three were analysed during intra-operative frozen section and no tumour was detected. This number is slightly lower than the average reported yield in the literature [27], but was based on the same anatomical dissection. The node that was missed may have been outside the normal template or may have been missed at 
the time of the SML, resulting in a late recurrence. Of the six patients from Group C who opted for clinical surveillance, none had disease recurrence after a mean follow-up of 37 months. Two studies have reported one case of non-visualization and found to have a metastatic lymph node [21, 27]. Based on our findings, we believe that patients with high risk factors such as G3 or $>\mathrm{pT} 1$ disease should be offered repeat DSNB with modified SML in the same setting, if a second non-visualization occurs, while patients with $<\mathrm{pT} 2$ stage and $<\mathrm{G} 3$ grade disease could be offered close clinical and ultrasound surveillance according to well-defined protocols. A further prospective study using the protocol defined above is planned to confirm its validity.

There are several limitations to this study. Its retrospective nature implies the potential for information and selection bias. Secondly, although the cohort of patients who underwent DSNB was large, the number of nonvisualizations was still too small to allow statistical analysis to be carried out on predictors of nonvisualization.

In conclusion, patients who have unilateral nonvisualization after DSNB have a high probability (86\%) of successful visualization during a repeat procedure. A riskadapted approach is proposed in which clinical surveillance is an alternative to repeat DSNB in the absence of risk factors. We recommend the latter strategy only to be applied in patients harbouring primary stage $<$ pT2 and grade $<$ G3 disease, whilst acknowledging that this is based on three patients in our study and further investigation is required to confirm this.

\section{Conflict of Interest}

None declared.

\section{Acknowledgement}

This study was supported by the National Institute for 
Health Research University College London Hospitals

Biomedical Research Centre.

\section{Abbreviations}

\section{DSNB}

dynamic sentinel lymph node biopsy

SML

superficial modified inguinal lymphadenectomy

SPECT

single-photon emission CT

1 Backes DM, Kurman RJ, Pimenta JM, Smith JS.

Systematic review of human papillomavirus prevalence in invasive penile cancer. Cancer Causes Control 2009; 20: 449-57

2 Chaux A, Netto GJ, Rodriguez IM et al. Epidemiologic profile, sexual history, pathologic features, and human papillomavirus status of 103 patients with penile carcinoma. World J Urol 2013; 31: 861-7

3 Shabbir M, Muneer A, Kalsi J et al. Glans resurfacing for the treatment of carcinoma in situ of the penis: surgical technique and outcomes. Eur Urol 2011; 59: 142-7

4 Hakenberg OW, Comperat EM, Minhas S et al. EAU guidelines on penile cancer: 2014 update. Eur Urol 2015; 67: 142-50

5 Horenblas S, van Tinteren $\mathrm{H}$. Squamous cell carcinoma of the penis. IV. Prognostic factors of survival: analysis of tumor, nodes and metastasis classification system. J Urol 1994; 151: 1239-43

6 Jemal A, Siegel R, Ward E, Murray T, Xu J, Thun MJ. Cancer statistics, 2007. CA Cancer J Clin 2007; 57: 43-66

7 Lont AP, Kroon BK, Gallee MP, van Tinteren H, Moonen 
LM, Horenblas S. Pelvic lymph node dissection for penile carcinoma: extent of inguinal lymph node involvement as an indicator for pelvic lymph node involvement and survival. $J$ Urol 2007; 177: 947-52

8 Pandey D, Mahajan V, Kannan RR. Prognostic factors in node-positive carcinoma of the penis. J Surg Oncol 2006; 93: 133-8

9 Parkin DM, Muir CS. Cancer incidence in five continents. Comparability and quality of data. IARC Sci Publ 1992; 120: 45-173

10 Ravi R. Correlation between the extent of nodal involvement and survival following groin dissection for carcinoma of the penis. Br J Urol 1993; 72 (5 Pt 2): 817-9

11 Sanchez-Ortiz RF, Pettaway CA. The role of lymphadenectomy in penile cancer. Urol Oncol 2004; 22: 236-44

12 Srinivas V, Morse MJ, Herr HW, Sogani PC, Whitmore WF Jr. Penile cancer: relation of extent of nodal metastasis to survival. J Urol 1987; 137: 880-2

13 Horenblas S. Lymphadenectomy for squamous cell carcinoma of the penis. Part 2: the role and technique of lymph node dissection. BJU Int 2001; 88: 473-83

14 Bevan-Thomas R, Slaton JW, Pettaway CA. Contemporary morbidity from lymphadenectomy for penile squamous cell carcinoma: the M.D. Anderson Cancer Center Experience. J Urol 2002; 167: 1638-42

15 Cabanas RM. An approach for the treatment of penile carcinoma. Cancer 1977; 39: 456-66

16 Leijte JA, Kroon BK, Valdes Olmos RA, Nieweg OE, Horenblas S. Reliability and safety of current dynamic sentinel node biopsy for penile carcinoma. Eur Urol 2007; 52: 170-7 
17 Leijte JA, Hughes B, Graafland NM et al. Two-center evaluation of dynamic sentinel node biopsy for squamous cell carcinoma of the penis. J Clin Oncol 2009; 27: 3325-9

18 Kroon BK, Lont AP, Valdes Olmos RA, Nieweg OE, Horenblas S. Morbidity of dynamic sentinel node biopsy in penile carcinoma. J Urol 2005a; 173: 813-5

19 Omorphos S, Saad Z, Arya M et al. Feasibility of performing dynamic sentinel lymph node biopsy as a delayed procedure in penile cancer. World J Urol 2016; 34: 329-35

20 Kroon BK, Horenblas S, Meinhardt W et al. Dynamic sentinel node biopsy in penile carcinoma: evaluation of 10 years experience. Eur Urol 2005b; 47: 601-6

21Kroon BK, Valdes Olmos R, Nieweg OE, Horenblas S. Non-visualization of sentinel lymph nodes in penile carcinoma. Eur J Nucl Med Mol Imaging 2005c; 32: 1096-9

22 Birdwell RL, Smith KL, Betts BJ, Ikeda DM, Strauss HW, Jeffrey SS. Breast cancer: variables affecting sentinel lymph node visualization at preoperative lymphoscintigraphy. Radiology 2001; 220: 47-53

23 Brenot-Rossi I, Houvenaeghel G, Jacquemier J et al. Nonvisualization of axillary sentinel node during lymphoscintigraphy: is there a pathologic significance in breast cancer? J Nucl Med 2003; 44: 1232-7

24 Krausz Y, Ikeda DM, Jadvar H, Langleben D, Birdwell R, Strauss HW. Non-visualization of sentinel lymph node in patients with breast cancer. Nucl Med Commun 2001; 22: 25-32

25 Moffat FL Jr, Gulec SA, Sittler SY et al. Unfiltered sulfur colloid and sentinel node biopsy for breast cancer: technical and kinetic considerations. Ann Surg Oncol 1999; 6: 746-55

26 Tanis PJ, van Sandick JW, Nieweg OE et al. The hidden 
sentinel node in breast cancer. Eur J Nucl Med Mol Imaging 2002; 29: 305-11

27 Kirrander P, Andren O, Windahl T. Dynamic sentinel node biopsy in penile cancer: initial experiences at a Swedish referral centre. BJU Int 2013; 111 (3 Pt B): E48-53 